\title{
HURRICANE WATER LEVEL PREDICTION USING SURROGATE MODELING
}

\author{
Jeffrey A. Melby, Noble Consultants-G.E.C. Inc., jmelby@nobleconsultants.com \\ Fatima Diop, Noble Consultants-G.E.C. Inc. \\ Norberto Nadal-Caraballo, U.S. Army Engineer R\&D Center \\ Alex Taflanidis, Notre Dame University \\ Victor Gonzalez, U.S. Army Engineer R\&D Center
}

\section{SURROGATE APPROACH}

Artificial intelligence (Al) technologies are being exploited to solve many coastal engineering problems. In particular, artificial neural networks and surrogate models are used for forecasting, Monte Carlo simulation, data assimilation, and empirical simulation, among others. The primary requirements are that the training data characterize the underlying phenomena and span the range of parameter space for the processes being modelled. Unfortunately, significant resources are often required to develop a training set that accurately reflects natural phenomenon. This is the case for developing a hurricane response model that accurately predicts storm response for all possible future hurricanes.

One of the more exciting $\mathrm{Al}$ areas of application is in integrated surrogate and process model solutions. In this class of applications, the parameter space is initially approximated using statistical methods, like the joint probability method, design of experiments, or heuristic arguments yielding a subset of parameter combinations that crudely approximates the final desired model. A crude subset of simulations is then built using the process model (e.g. numerical, physical, analytical). Then a preliminary surrogate model is trained and this surrogate is used to populate the next increment of parameter space very efficiently using a global optimization scheme to define the optimal incremental parameter set. The process is repeated until a target error metric is achieved. The methods can be applied for varied levels of desired fidelity, and resource demands, from crude surrogates for reconnaissance level studies to highly accurate surrogates.

For this study, the surrogate was constructed using kriging (Jia et al. 2015). The high fidelity coupled surge and wave numerical modelling for the Gulf of Mexico was used as the training set. The numerical model was either ADCIRC and STWAVE or ADCIRC and SWAN in the nearshore. The surrogate models were trained using tropical storm parameters (latitude, longitude, central pressure, radius to maximum wind speed, storm heading, and forward speed) at a specific location as inputs and individual responses (e.g. surge) as outputs. Tide was computed separately using ADCIRC and linearly superimposed with surge to get total water level. The regional surrogates accurately reproduced both peaks and time series of water levels for historical storms. An extensive validation was conducted to determine the optimal application of the kriging approach. In this paper we will report the efficient design-of-experiments approach, surrogate training and validation.

\section{SOME RESULTS}

The kriging model was applied for Hurricane Harvey that struck the Texas coast in August of 2017. The catastrophic storm flooding was primarily a result of rainfall but there was significant storm surge in the landfalling region. The kriging model was trained for 2 Texas regions and used to predict total water level for Harvey. The model was initially applied at NOAA Advisory 16, just after the storm spun up to hurricane intensity and 36 hours before landfall. The model run time is less than 1 second. Figures $1 \mathrm{a}$ and $1 \mathrm{~b}$ show the water level compared to 2 gages for the prediction at Advisory 16 , just after the storm intensified to hurricane strength and 36 hours before landfall. Generally the model predicts the pre-peak and peak water levels well but not the infilling portion after landfall.

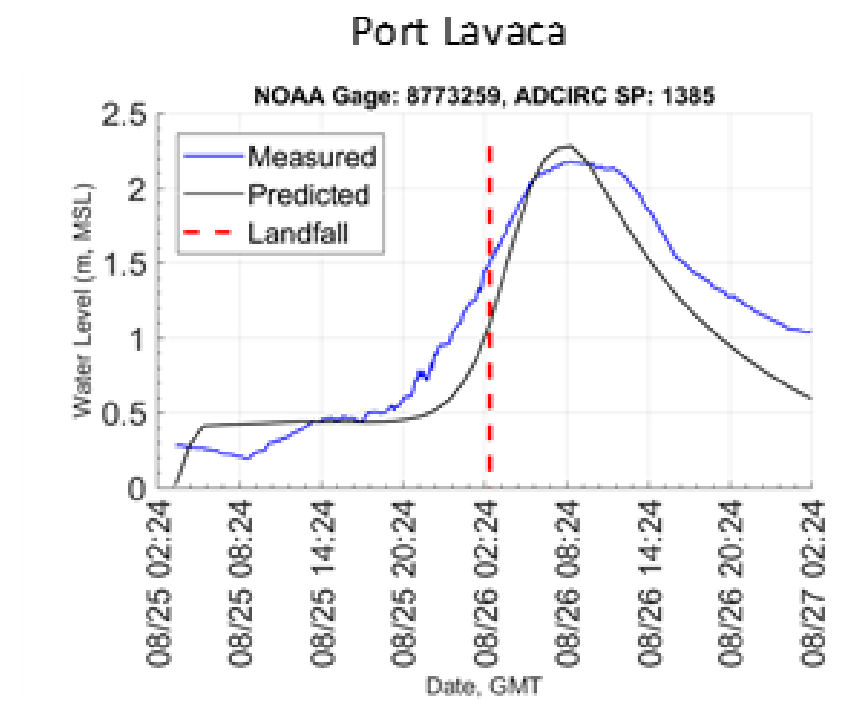

Figure 1a. Surrogate predicted and measured water level at Port Lavaca gage at Advisory 16, 36 hours before landfall. 


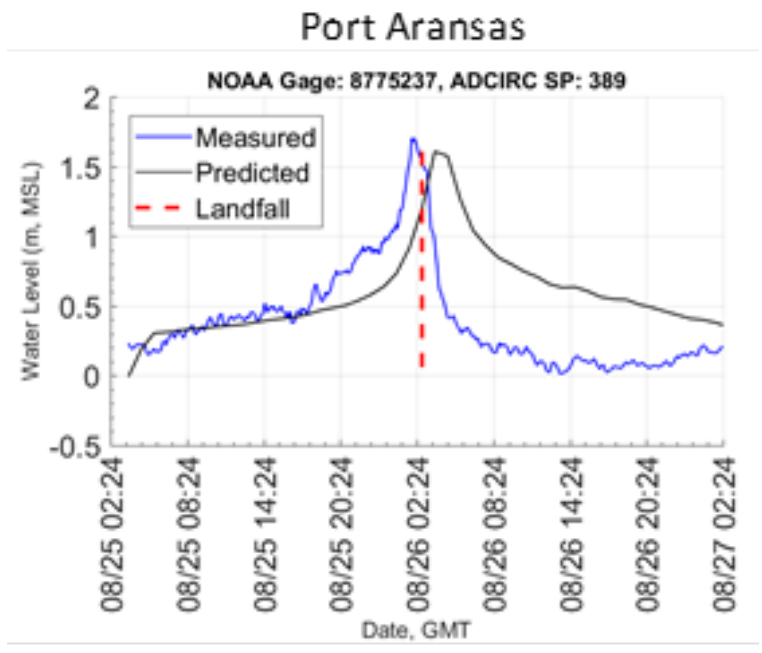

Figure $1 \mathrm{~b}$. Surrogate predicted and measured water level at Port Aransas gage at Advisory 16, 36 hours before landfall.

\section{REFERENCES}

Jia, G., Taflanidis, A.A., Nadal-Caraballo, N.C., Melby, J.A., Kennedy, A.B., Smith, J.M., (2015). "Surrogate modelling for storm surge prediction using an existing database of synthetic storms; addressing time-dependence of output and implementation over an

extended coastal region," Natural Hazards 81:909-938. 